\title{
Comparative benefits and harms of second generation antidepressants and cognitive behavioral therapies in initial treatment of major depressive disorder: systematic review and meta-analysis
}

\author{
Halle R Amick, ${ }^{1}$ Gerald Gartlehner, ${ }^{2,3}$ Bradley N Gaynes, ${ }^{4}$ Catherine Forneris, ${ }^{4}$ Gary N Asher, ${ }^{5}$ \\ Laura C Morgan, ${ }^{2}$ Emmanuel Coker-Schwimmer, ${ }^{1}$ Erin Boland, ${ }^{2}$ Linda J Lux, ${ }^{2}$ Susan Gaylord, ${ }^{6}$ \\ Carla Bann, ${ }^{2}$ Christiane Barbara Pierl, ${ }^{3}$ Kathleen N Lohr ${ }^{2}$
}

Theps Center for Health Services Research, University of North Carolina at Chapel Hill,

725 Martin Luther King Jr Boulevard, Chapel Hill, NC 27599, USA

${ }^{2}$ RTI International, 3040 Cornwallis Road, Research Triangle Park, NC 27709, USA ${ }^{3}$ Department for Evidencebased Medicine and Clinical Epidemiology, Danube University, 3500 Krems, Austria ${ }^{4}$ Department of Psychiatry, University of North Carolina at Chapel Hill, 101 Manning Drive, Chapel Hill, NC 27599, USA ${ }^{5}$ Department of Family Medicine, University of North Carolina at Chapel Hill, 590 Manning Drive, Chapel Hill, NC 27599, USA

${ }^{6}$ Department of Physical Medicine and Rehabilitation, University of North Carolina at Chapel Hill, 101 Manning Drive, Chapel Hill, NC 27599, USA

Correspondence to: H R Amickamick@med.unc.edu

Additional material is published online only. To view please visit the journal online (http://dx.doi. org/10.1136/bmj.h6019)

Cite this as: BMJ 2015;351:h6019 doi: 10.1136/bmj.h6019

Accepted: 24 Oct 2015

\section{ABSTRACT}

\section{STUDY QUESTION}

What are the benefits and harms of second generation antidepressants and cognitive behavioral therapies (CBTs) in the initial treatment of a current episode of major depressive disorder in adults?

\section{METHODS}

This was a systematic review including qualitative assessment and meta-analyses using random and fixed effects models. Medline, Embase, the Cochrane Library, the Allied and Complementary Medicine Database, PsycINFO, and the Cumulative Index to Nursing and Allied Health Literature were searched from January1990 through January 2015. The 11 randomized controlled trials included compared a second generation antidepressant CBT. Ten trials compared antidepressant monotherapy with CBT alone; three compared antidepressant monotherapy with antidepressant plus CBT.

\section{SUMMARY ANSWER AND LIMITATIONS}

Meta-analyses found no statistically significant difference in effectiveness between second generation antidepressants and CBT for response (risk ratio 0.91, 0.77 to 1.07$)$, remission $(0.98,0.73$ to 1.32$)$, or change in 17 item Hamilton Rating Scale for Depression score (weighted mean difference, $-0.38,-2.87$ to 2.10). Similarly, no significant differences were found in rates of overall study discontinuation (risk ratio 0.90, 0.49 to 1.65) or discontinuation attributable to lack of efficacy $(0.40,0.05$ to 2.91$)$. Although more patients treated with a second generation antidepressant than receiving CBT withdrew from studies because of

\section{WHAT IS ALREADY KNOWN ON THIS TOPIC}

Second generation antidepressants and cognitive behavioral therapy have evidence bases of benefits and harms in major depressive disorder

However, primary care physicians require high quality evidence of the comparative effectiveness of the available treatments to select and manage the best options for their patients

\section{WHAT THIS STUDY ADDS}

The available evidence suggests no difference in treatment effects of second generation antidepressants and cognitive behavioral therapies, either alone or in combination, in major depressive disorder

Given that patients may have personal preferences for one first line treatment over the other, both treatments should be made accessible, either alone or in combination, to primary care patients with major depressive disorder

adverse events, the difference was not statistically significant (risk ratio 3.29, 0.42 to 25.72). No conclusions could be drawn about other outcomes because of lack of evidence. Results should be interpreted cautiously given the low strength of evidence for most outcomes. The scope of this review was limited to trials that enrolled adult patients with major depressive disorder and compared a second generation antidepressant with CBT, and many of the included trials had methodological shortcomings that may limit confidence in some of the findings.

WHAT THIS STUDY ADDS

Second generation antidepressants and CBT have evidence bases of benefits and harms in major depressive disorder. Available evidence suggests no difference in treatment effects of second generation antidepressants and CBT, either alone or in combination, although small numbers may preclude detection of small but clinically meaningful differences.

FUNDING, COMPETING INTERESTS, DATA SHARING This project was funded under contract from the Agency for Healthcare Research and Quality by the RTI-UNC Evidence-based Practice Center. Detailed methods and additional information are available in the full report, available at http://effectivehealthcare.ahrq.gov/.

\section{Introduction}

Major depressive disorder is the most prevalent and disabling form of depression, affecting more than 32 million Americans. ${ }^{1}$ In any given year, nearly $7 \%$ of the American adult population has an episode of major depressive disorder, but only about half of these people seek care. ${ }^{1}$ For patients who do obtain care, only $20 \%$ receive adequate treatment. ${ }^{1}$ Based on available evidence based guidelines, this would include either drug therapy (at least two months of an appropriate drug for major depressive disorder plus more than four visits to any type of physician) or psychotherapy (at least eight visits with any healthcare professional lasting an average of at least 30 minutes)..$^{2-4}$

Treatment for major depressive disorder is often started in a primary care setting, ${ }^{5}$ and patients generally receive drugs as the standard treatment. ${ }^{6}$ Of the available antidepressants, second generation antidepressants are the most commonly prescribed agents. ${ }^{6}$ These drugs include selective serotonin reuptake inhibitors, serotonin and norepinephrine reuptake 
inhibitors, and other drugs with related mechanisms of action that target specific neurotransmitters. Although these drugs have different mechanisms of action, evidence suggests that no substantial differences in benefits exist among them as a class. ${ }^{7}$

Although drug therapy is the treatment most frequently offered to depressed primary care patients, data from studies of antidepressant drugs show that approximately $20 \%$ of patients do not fill their prescriptions. Moreover, even if they start a course of treatment, they may discontinue early before receiving an adequate course. ${ }^{8}$ One reason for lack of adherence to second generation antidepressants is the frequency and severity of side effects. More than $60 \%$ of patients have at least one adverse effect during treatment with a second generation antidepressant. Although most adverse effects are minor (for example, constipation, diarrhea, and dizziness), they frequently lead to discontinuation of treatment. ${ }^{9}$

Patients' preferences for starting or switching treatment may also play a key role in acceptance and continuation of treatment for major depressive disorder. Some research suggests that patients might prefer treatment with psychotherapy over drugs. ${ }^{10-14}$ Reasons for preferring psychotherapy over drugs include concerns about side effects and perceived "addictiveness" of drugs. ${ }^{1215-18}$ In addition, women and ethnic minorities may be more likely to prefer psychotherapies over drugs..$^{12131920}$ Regardless of which treatment patients prefer, some evidence suggests that patients who receive their treatment of choice fare better than those whose treatment is incongruous with their preferences. ${ }^{1121}$

In general, psychotherapeutic interventions aim to help patients to identify how past and present factors may contribute to their depression and to teach them how to deal effectively with them. ${ }^{22-25}$ Cognitive behavioral therapy is based on a combination of basic behavioral and cognitive principles. Briefly, it helps patients to understand and examine how their thoughts, moods, and behaviors interact in a way that can result in or worsen depression. Patients are taught how to replace dysfunctional thoughts and behaviors with more adaptive ones, which can reduce distress and improve mood.

Given the range of available treatments for patients with major depressive disorder, each with its own evidence base of benefits and harms, primary care physicians require high quality evidence of the comparative effectiveness of the available treatments to select and manage the best options for their patients. This paper focuses on the comparative benefits of second generation antidepressants and cognitive behavioral therapies as an initial treatment of a current episode of major depressive disorder in adults. Our results come from a larger comparative effectiveness review of benefits and harms of second generation antidepressants, psychotherapies (including psychotherapies other than cognitive behavioral therapy), complementary and alternative medicine treatments, and exercise interventions for major depressive disorder funded by the US Agency for Healthcare Research and Quality. ${ }^{26}$

\section{Methods}

Detailed methods are available in the full report. ${ }^{26}$ The full search strategy is available in web appendix A. In brief, we searched Medline, Embase, the Cochrane Library, the Allied and Complementary Medicine Database, PsycINFO, and the Cumulative Index to Nursing and Allied Health Literature for randomized and non-randomized controlled trials published from January1990 through January 2015. For drugs, we searched for individual generic drug names and broader second generation antidepressant related terms; for cognitive behavioral therapy studies, we used the terms "psychotherapy," "cognitive therapy," and "cognitive behavioral therapy" in titles or abstracts. To detect unpublished studies, we searched ClinicalTrials.gov, the World Health Organization's International Clinical Trials Registry Platform, Drugs@FDA, the European Medicines Agency, the National Institute of Mental Health website, the American Psychological Association website, Scopus, the Conference Proceedings Citation Index, and reference lists of pertinent reviews and included trials.

Table 1 contains the eligibility criteria that we applied to search results. Briefly, we included studies that compared a second generation antidepressant with cognitive behavioral therapy for the initial treatment of a current episode of major depressive disorder in adults. Cognitive behavioral therapy is the umbrella "class" with which we compared second generation antidepressants. However, we recognize the challenge in organizing and categorizing psychological interventions in systematic reviews. In an effort to enhance the consistency of categorization of psychotherapies in this

\footnotetext{
Table 1 Inclusion criteria for studies comparing second generation antidepressant with cognitive behavioral therapy

\begin{tabular}{|c|c|}
\hline Parameter & Criteria \\
\hline Population & Adult (18 years or older) outpatients with major depressive disorder during initial treatment attempt for current episode \\
\hline \multirow[t]{2}{*}{ Interventions } & $\begin{array}{l}\text { Second generation antidepressants approved for treatment of major depressive disorder by the US Food and Drug Administration: bupropion, citalopram, } \\
\text { desvenlafaxine, duloxetine, fluoxetine, escitalopram, fluvoxamine, levomilnacipran, mirtazapine, nefazodone, paroxetine, sertraline, trazodone, venlafaxine, } \\
\text { vilazodone, vortioxetine }\end{array}$ \\
\hline & Cognitive behavioral therapies as classified by Cochrane Collaborative Depression, Anxiety and Neurosis Group's framework ${ }^{27}$ \\
\hline \multirow[t]{2}{*}{ Outcomes } & $\begin{array}{l}\text { Benefits: response, remission, speed of response, speed of remission, relapse, quality of life, functional capacity, suicidal ideas or behaviors, hospital } \\
\text { admission }\end{array}$ \\
\hline & $\begin{array}{l}\text { Harms: overall adverse events, withdrawals because of adverse events, serious adverse events, specific adverse events (including hyponatremia, seizures, } \\
\text { suicidal ideas or behaviors, hepatotoxicity, weight gain, gastrointestinal symptoms, sexual side effects), withdrawals because of specific adverse events }\end{array}$ \\
\hline \multirow[t]{2}{*}{ Study designs } & Benefits: randomized controlled trials, systematic reviews, and meta-analyses \\
\hline & $\begin{array}{l}\text { In addition for harms: non-randomized controlled trials, prospective controlled cohort studies, retrospective controlled cohort studies, case-control studies, } \\
\text { all with minimum sample size of } 500 \text { participants }\end{array}$ \\
\hline
\end{tabular}
}


review and our ability to compare our findings with those of other large reviews, we have used the Cochrane Collaborative Depression, Anxiety and Neurosis Group's framework for categorizing psychological interventions. ${ }^{27}$ In addition to the umbrella term cognitive behavioral therapy, the framework's cognitive behavioral therapy category includes problem solving therapy, rational emotive therapy, reality therapy, restructuring, role play, schemas, self control, and stress management. If the authors of an included trial specified the particular form of the behavioral intervention, we mention it; if they did not, we use the term cognitive behavioral therapy.

Two trained team members independently reviewed all abstracts and full text articles against predefined inclusion/exclusion criteria. Investigators resolved disagreements about inclusion or exclusion by consensus or by involving a third reviewer. We designed, pilot tested, and used a structured data abstraction form to ensure consistency of data abstraction. Trained reviewers initially abstracted data from each study; a senior reviewer evaluated the completeness and accuracy of the data abstraction.

We rated the risk of bias for each relevant outcome of a study as low, moderate, or high. To determine risk of bias in a standardized way, we used the Cochrane Risk of Bias tool to appraise randomized controlled trials. ${ }^{28}$ Two independent reviewers assigned ratings for risk of bias. They resolved any disagreements by discussion and consensus or by consulting a third member of the team. We included all eligible studies in this review regardless of risk of bias, but we used studies rated as at high risk of bias only in sensitivity analyses. Risk of bias assessments are shown in appendix B.

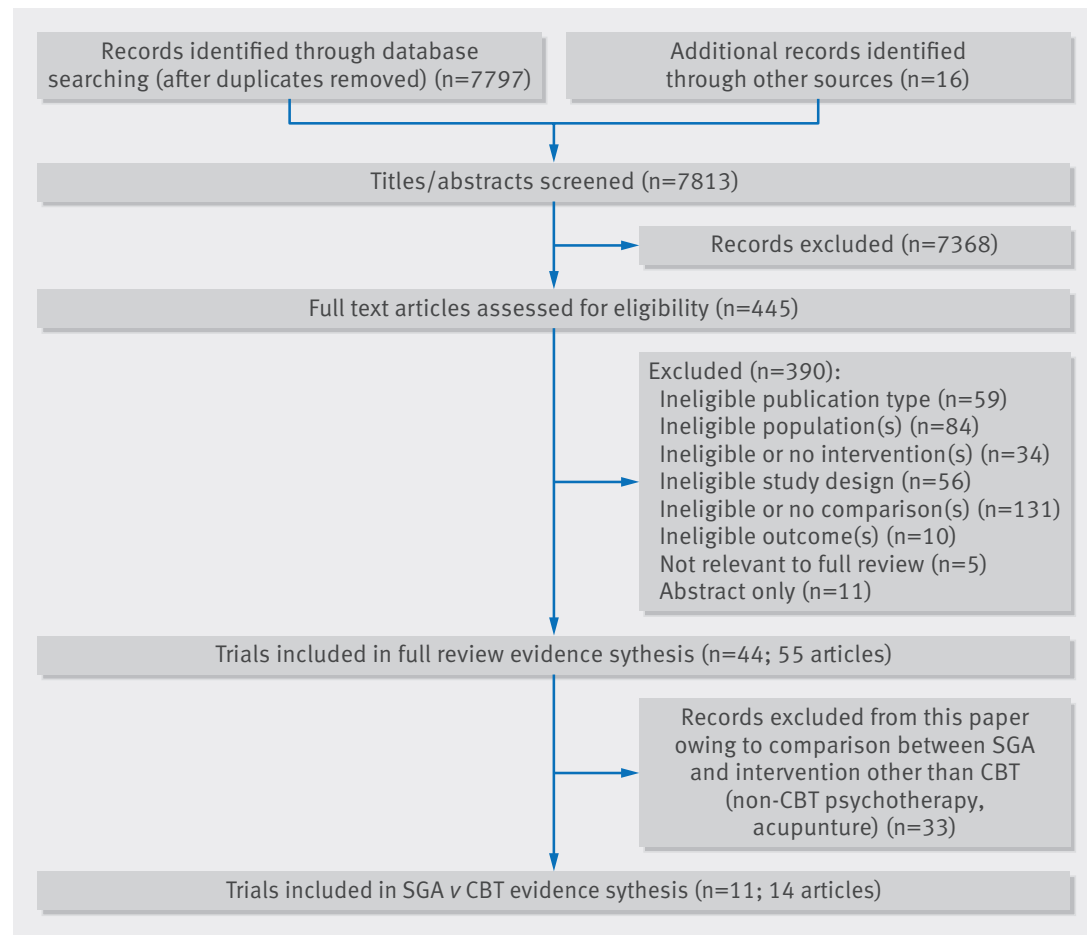

Fig 1 | PRISMA diagram for second generation antidepressants versus cognitive behavioral therapy in treatment of major depressive disorders
We graded the strength of evidence on the basis of guidance established by the US Agency for Healthcare Research and Quality for the Evidence-based Practice Center program..$^{29}$ This approach, which is based largely on the approach developed by the Grading of Recommendations Assessment, Development and Evaluation (GRADE) Working Group, incorporates five domains: study limitations, consistency, directness, precision, and reporting bias. Grades (high, moderate, low, insufficient) reflect the confidence we had about the evidence for a specific outcome on the comparative benefits and harms of the interventions.

Evidence suggests that no substantial differences in benefits exist among the different types of second generation antidepressants ${ }^{7}$; therefore, in all meta-analyses we compared second generation antidepressants as a class with cognitive behavioral therapies. When we did meta-analyses, we assessed statistical heterogeneity in effects between studies by calculating the $\chi^{2}$ statistic and Cochran's q. We used the $\mathrm{I}^{2}$ statistic (the proportion of variation in study estimates attributable to heterogeneity) to estimate the magnitude of heterogeneity. We examined potential sources of heterogeneity by using sensitivity and subgroup analyses.

For all analyses, we used random effects and fixed effects models to estimate comparative effects. We used DerSimonian and Laird models for random effects analyses. For studies with attrition, we used a "worst case" assumption that non-completers failed to respond or remit. We used funnel plots to assess publication bias, but given the small number of studies in our meta-analyses, these tests have low sensitivity to detect publication bias. We used Comprehensive Meta-analysis, version 3.2 (Biostat; Englewood, NJ, USA) for all meta-analyses.

\section{Patient involvement}

A representative of the National (US) Board of Directors of the National Alliance on Mental Illness participated in the refinement of the research topic and the development of the preliminary research questions and review criteria. The research question and a draft version of the report were posted online for input from all members of the public, including patients and their advocates. Dissemination of results will include a translation of the results into short, easy to read summaries and tools that can be used by patients, caregivers, and others who use healthcare services.

\section{Results}

\section{Literature searches and evidence base}

Our searches for the full report identified 7813 citations, of which we included 11 primary randomized controlled trials (reported in 14 articles) that compared a second generation antidepressant with cognitive behavioral therapy and provided data relevant to this paper (fig 1). ${ }^{30-43}$ Those studies provided information on 1511 patients with major depressive disorder.

Two trials were conducted in primary care settings ${ }^{3839}$; the remainder took place in outpatient mental healthcare locations. Four trials took place solely in the 
United States; 30353738 other countries included Canada, ${ }^{33} 3642$ England, ${ }^{39}$ Germany, ${ }^{40}$ Iran, ${ }^{43}$ and Romania. ${ }^{32}$

Generally, patients were aged between 18 and 65 years; most trials reported a mean age between 35 and 45 years. In all trials, most patients were female. One trial enrolled only women. ${ }^{38}$ In the few trials that reported race or ethnicity, a quarter of patients were non-white. All trials reported mean baseline severity of depression of at least a moderate degree; most trials reported mean baseline scores on the 17 item Hamilton Rating Scale for Depression between 16 (moderate depression) and 23 (severe). The total daily dose of each second generation antidepressant drug was within the usual ranges prescribed for adults.

Table 2 describes the 11 included trials (14 publications) of a second generation antidepressant compared with cognitive behavioral therapy. Second generation antidepressants used in the trials were fluoxetine, ${ }^{32} \mathrm{flu}$ voxamine, ${ }^{39}$ paroxetine, ${ }^{35-3739}$ sertraline, ${ }^{3640}$ venlafaxine, ${ }^{33} 36$ citalopram, ${ }^{43}$ and escitalopram..$^{30}{ }^{42}$ Because of the inherent heterogeneity in delivery of psychotherapies, even for those under the umbrella of cognitive behavioral therapy, we used the individual studies' definitions of the cognitive therapies provided. Six trials used cognitive behavioral therapy, 303336384042 four used the specific cognitive therapy modality, 32353743 and one each used problem solving therapy and rational emotive therapy modalities. ${ }^{32} 39$ Trial counts exceed 11 because one trial had both cognitive therapy and rational emotive therapy arms. ${ }^{32}$ All but one trial compared second generation antidepressant monotherapy with cognitive behavioral therapy alone; Lam and colleagues compared second generation antidepressant monotherapy with second generation antidepressant plus cognitive behavioral therapy. ${ }^{42}$ Two trials included an additional comparison of second generation antidepressant monotherapy with a combination of second generation antidepressant and cognitive behavioral therapy. ${ }^{39} 43$ Treatment duration ranged from eight weeks to one year; some trials also reported follow-up results once patients were no longer receiving active treatments.

We present our findings first by treatment comparison (monotherapy comparisons followed by combination therapy comparisons). Within each comparison, we present the benefits followed by the harms. Table 3 summarizes the findings and respective strength of evidence ratings for all comparisons and outcomes.

Second generation antidepressant versus cognitive behavioral therapy: monotherapy comparisons

We did random effects meta-analyses of trials rated as at low or medium risk of bias for remission, response, and change in 17 item Hamilton Rating Scale for Depression score. We also did sensitivity analyses for those outcomes including additional trials rated as at high risk of bias. ${ }^{303336}$

For remission, we included three trials (four comparisons) with a total of 432 patients. ${ }^{32} 3539$ We analyzed results measured between 12 and 16 weeks. The three trials defined remission on the basis of a 17 item
Hamilton Rating Scale for Depression score of either less than $7^{32}$ or less than or equal to $7 .^{35} 39$ One trial also required a score less than or equal to 10 on the Beck Depression Inventory for remission. ${ }^{35}$ Patients treated with second generation antidepressants had numerically lower but not significantly different remission rates than patients receiving cognitive behavioral therapy $(40.7 \% \vee 47.9 \%$; risk ratio 0.98, 95\% confidence interval 0.73 to 1.32; fig 2 ; low strength of evidence). Our sensitivity analysis including three trials at high risk of bias yielded a similar, non-significant difference (risk ratio $1.08,0.90$ to 1.29$).{ }^{3033} 36$

For response, we included results from 660 patients studied in five trials (six comparisons). ${ }^{32} 35373940$ We analyzed results measured between eight and 16 weeks. The five trials defined response as a $50 \%$ or greater reduction from baseline in 17 item Hamilton Rating Scale for Depression score. Treatment effects were similar for second generation antidepressants and cognitive behavioral therapies $(44.2 \% v 45.5 \%$; risk ratio 0.91 , 0.77 to 1.07; fig 3; moderate strength of evidence). The sensitivity analysis including three studies at high risk of bias yielded a similarly non-statistically significant difference in response between second generation antidepressants and cognitive behavioral therapies (risk ratio $1.02,0.83$ to 1.25$).{ }^{303336}$

Our analysis of the two trials (three comparisons; 249 patients) $)^{32} 39$ that reported change in 17 item Hamilton Rating Scale for Depression scores at eight weeks or longer found no statistically significant difference between second generation antidepressants and cognitive behavioral therapies (weighted mean difference -0.38 , -2.87 to 2.10; fig 4). Adding the trials at high risk of bias to the model yielded no difference in comparative effectiveness $(-0.27,-2.44$ to 1.90$)$. $^{3340}$

Two trials, both rated as at medium risk of bias, reported response, remission, or change in 17 item Hamilton Rating Scale for Depression score at time points beyond 16 weeks. In one, ${ }^{32}$ patients receiving either rational emotive therapy or cognitive therapy reported higher rates of remission and response at six months than patients taking a second generation antidepressant, although neither difference was statistically significant. At six months, patients receiving rational emotive therapy or cognitive therapy reported significantly lower 17 item Hamilton Rating Scale for Depression scores than did the patients taking the second generation antidepressant. In the trial that compared second generation antidepressants with problem solving therapy, ${ }^{39}$ the rate of remission at one year was higher in the problem solving therapy arm, although rate of response at one year was higher in the second generation antidepressant arm. In that trial, patients' 17 item Hamilton Rating Scale for Depression scores continued to decline, with one year scores being lower in the problem solving therapy arm than the second generation antidepressant arm. Again, these differences failed to reach statistical significance.

With respect to other health outcomes, three trials reported relapse rates during off treatment follow-up. 32353645 Two trials defined relapse as symptom 


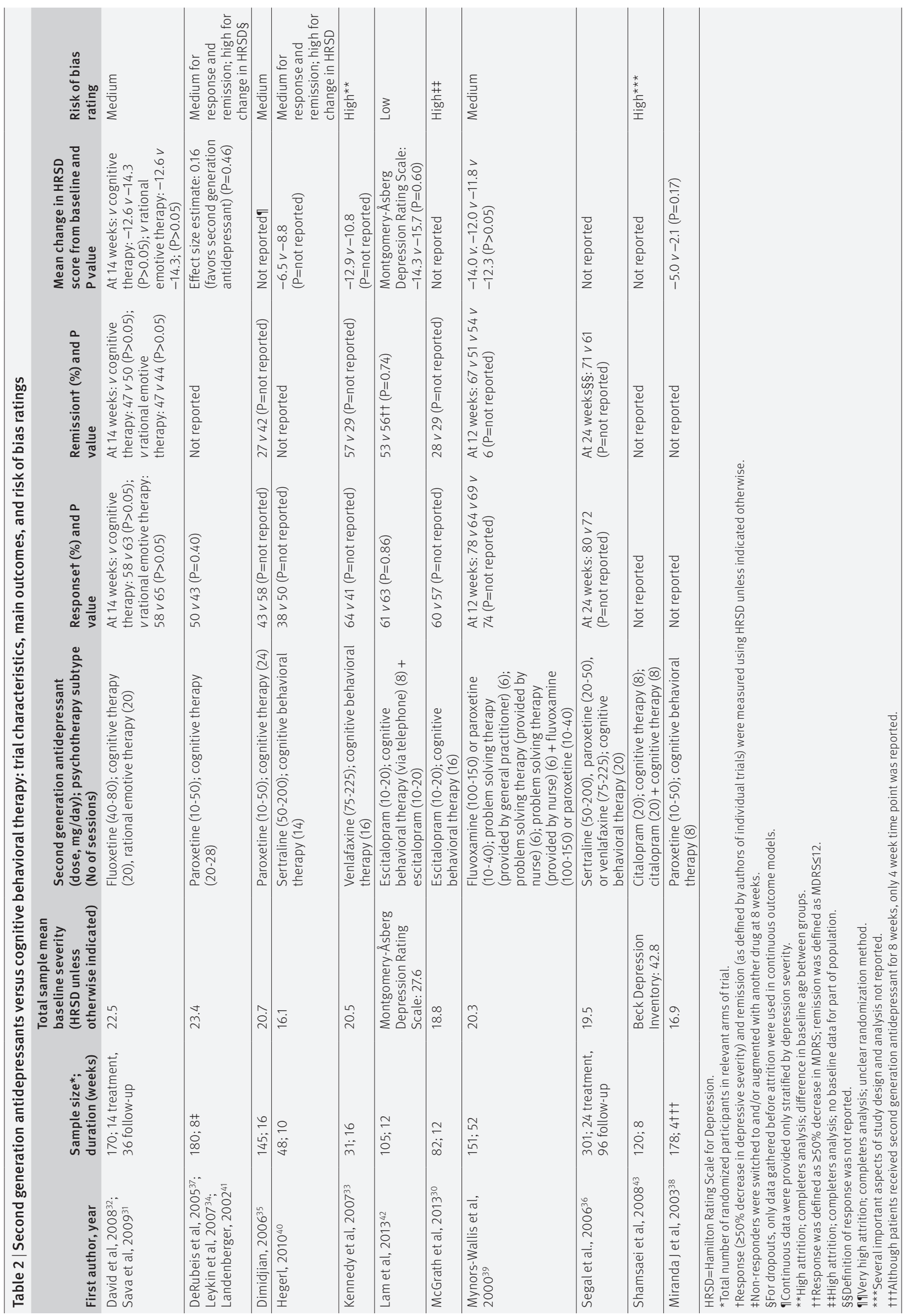




\begin{tabular}{|c|c|c|}
\hline $\begin{array}{l}\text { Comparison and outcome } \\
\text { of interest }\end{array}$ & $\begin{array}{l}\text { Strength of } \\
\text { evidence* }\end{array}$ & Findings \\
\hline \multicolumn{3}{|c|}{ Second generation antidepressant versus cognitive behavioral therapy monotherapy comparisons } \\
\hline Remission & Low & $\begin{array}{l}\text { Results from direct comparisons in three trials indicate that no differences in remission exist between second generation } \\
\text { antidepressants and cognitive behavioral therapy monotherapy (risk ratio } 0.98,95 \% \mathrm{Cl} 0.73 \text { to } 1.32 \text { ) }\end{array}$ \\
\hline Response & Moderate & $\begin{array}{l}\text { Results from direct comparisons in five trials indicate that no substantial differences in response exist between second } \\
\text { generation antidepressants and cognitive behavioral therapy monotherapy (risk ratio } 0.91,0.77 \text { to 1.07) }\end{array}$ \\
\hline Functional capacity & Low & $\begin{array}{l}\text { Results from one trial indicate that no substantial differences in functional capacity exist between second generation } \\
\text { antidepressants and cognitive behavioral therapy monotherapy }\end{array}$ \\
\hline Overall risk of adverse events & Insufficient & On the basis of one trial with few events, the evidence is insufficient to draw conclusions \\
\hline $\begin{array}{l}\text { Overall discontinuation of } \\
\text { treatment }\end{array}$ & Moderate & $\begin{array}{l}\text { Results from direct comparisons in four trials indicate that no significant differences exist in overall discontinuation } \\
\text { between patients treated with second generation antidepressants and those treated with cognitive behavioral therapy (risk } \\
\text { ratio } 1.00,0.55 \text { to 1.81) }\end{array}$ \\
\hline $\begin{array}{l}\text { Discontinuation of treatment } \\
\text { because of adverse events }\end{array}$ & Low & $\begin{array}{l}\text { Results from direct comparisons in three trials indicate that patients treated with second generation antidepressants have a } \\
\text { numerically but not statistically significant higher rate of discontinuation because of adverse events than those treated with } \\
\text { cognitive behavioral therapy (risk ratio } 2.54,0.39 \text { to } 16.47 \text { ) }\end{array}$ \\
\hline \multicolumn{3}{|c|}{ Second generation antidepressant versus combination of second generation antidepressant and cognitive behavioral therapy } \\
\hline Remission & Low & $\begin{array}{l}\text { Results from direct comparisons in two trials indicate that no substantial differences in remission exist between second } \\
\text { generation antidepressants and second generation antidepressants combined with cognitive behavioral therapy (risk ratio } \\
1.06,0.82 \text { to 1.38) }\end{array}$ \\
\hline Response & Low & $\begin{array}{l}\text { Results from direct comparisons in two trials indicate that no substantial differences in response exist between second } \\
\text { generation antidepressants and second generation antidepressants combined with cognitive behavioral therapy (risk ratio } \\
1.03,0.85 \text { to 1.26) }\end{array}$ \\
\hline Functional capacity & Low & $\begin{array}{l}\text { Results from one trial indicate that the combination of second generation antidepressant with cognitive behavioral therapy } \\
\text { results in statistically significantly greater improvement on } 3 / 4 \text { work functioning measures than second generation } \\
\text { antidepressant alone }\end{array}$ \\
\hline $\begin{array}{l}\text { Overall discontinuation of } \\
\text { treatment }\end{array}$ & Low & $\begin{array}{l}\text { Results from direct comparisons in two head to head trials indicate that no significant differences exist in overall } \\
\text { discontinuation between patients treated with second generation antidepressants and those treated with cognitive } \\
\text { behavioral therapy (risk ratio } 0.77,0.37 \text { to } 1.60 \text { ) }\end{array}$ \\
\hline $\begin{array}{l}\text { Discontinuation of treatment } \\
\text { because of adverse events }\end{array}$ & Low & $\begin{array}{l}\text { Results from direct comparisons in two head to head trials indicate that no significant differences exist in discontinuation } \\
\text { because of adverse events between patients treated with second generation antidepressants and those treated with } \\
\text { cognitive behavioral therapy (risk ratio } 2.93,0.72 \text { to } 11.91 \text { ) }\end{array}$ \\
\hline
\end{tabular}

*Grades (high, moderate, low, or insufficient) are based on methods guidance for US Agency for Healthcare Research and Quality for the Evidence-based Practice Center program. ${ }^{4} 4$

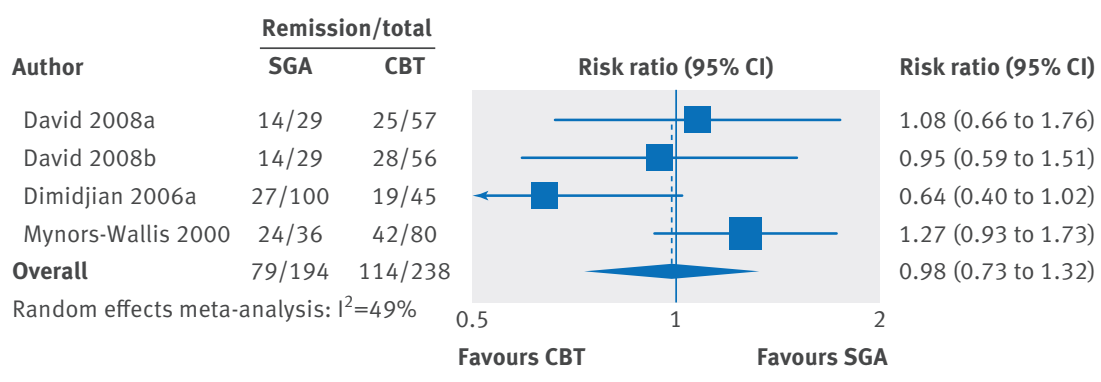

Fig 2 | Second generation antidepressants (SGA) versus cognitive behavioral therapy (CBT): remission

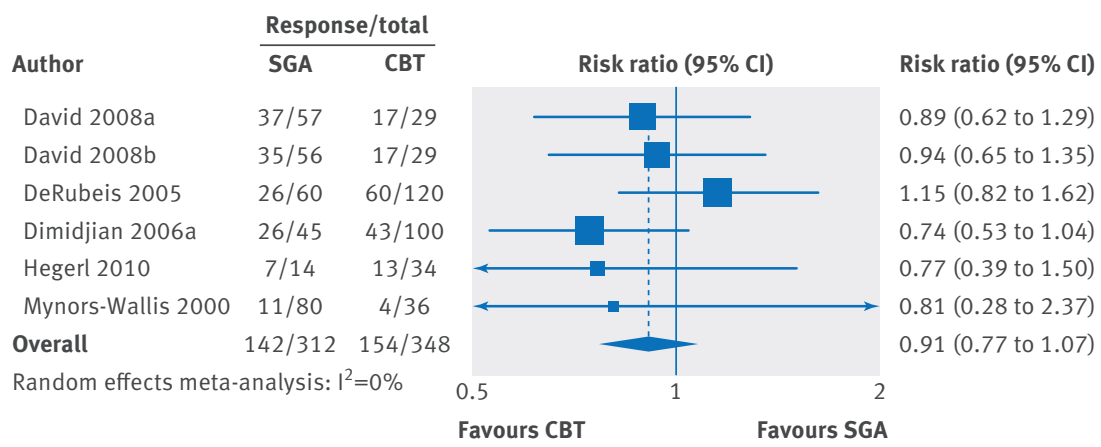

Fig 3 | Second generation antidepressants (SGA) versus cognitive behavioral therapy (CBT): response psychiatric status rating of 5 or greater during the first year of follow-up. ${ }^{35}$ During the follow-up period of that trial, ${ }^{35}$ patients who had initially received cognitive therapy did not receive any treatment, and patients who had received second generation antidepressant were randomized to continue second generation antidepressant or switched to a placebo pill.

In one trial at medium risk of bias, ${ }^{32} 10.6 \%$ of patients treated with second generation antidepressant relapsed within six months, compared with $2.1 \%$ and $6.1 \%$ of patients treated with rational emotive therapy and cognitive therapy, respectively (statistical significance not reported). In the other trial at medium risk of bias, ${ }^{35} 45$ the rates of relapse within the first year of follow-up were $39 \%$ for previous cognitive therapy, 53\% for patients who were in the second generation antidepressant arm and continued to receive it during follow-up, and 59\% for patients who received second generation antidepressants during the acute phase but were switched to placebo during follow-up. Previous cognitive therapy was significantly different from previous second generation antidepressant switched to placebo $(\mathrm{P}=0.02)$. In the trial rated as at high risk of bias, ${ }^{36} 47 \%$ of patients treated with a second generation antidepressant who achieved remission and 39\% of those treated with cognitive behavioral therapies who achieved remission relapsed within 18 months $(\mathrm{P}=0.40)$.

Finally, one trial at medium risk of bias reported recurrence during the second year of follow-up, defined as either a 17 item Hamilton Rating Scale for Depression levels meeting criteria for major depressive disorder; the third defined relapse as either a 17 item Hamilton Rating Scale for Depression score of 14 or greater or a 


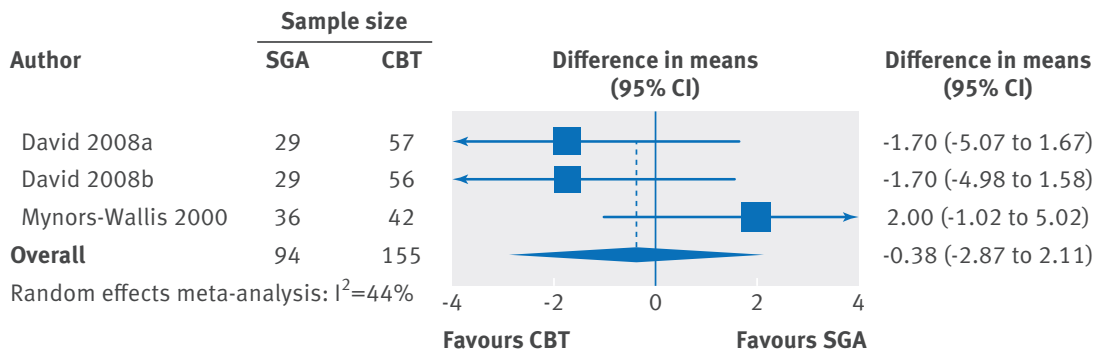

Fig 4 | Second generation antidepressants (SGA) versus cognitive behavioral therapy (CBT): change in 17 item Hamilton Rating Scale for Depression

score of 14 or greater or a psychiatric status rating of 5 or greater among those who did not relapse during year 1 of follow-up..$^{35}$ The rates of recurrence during year 2 were $24 \%$ for previous cognitive therapy and $52 \%$ for patients who were taking second generation antidepressants during the acute phase $(\mathrm{P}=0.06)$. Owing largely to small numbers of patients in each group (17 in each group), the difference was not statistically significant. The single trial (also at medium risk of bias) that reported measures of functional capacity used the Social Adjustment Scale ${ }^{39}$; second generation antidepressant and problem solving therapy did not differ at end of treatment or at 40 week off-treatment follow-up ( $\mathrm{P}>0.05$ at both times).

With regard to adverse events, reporting was generally poor, particularly for serious adverse events and specific adverse events. As a result, we analyzed rates of discontinuation as proxies for adverse events. Overall discontinuation from the four studies at medium risk of bias did not differ significantly between second generation antidepressants and cognitive behavioral therapies (risk ratio 1.00, 0.55 to $1.81 ; \mathrm{I}^{2}=47 \%$; moderate strength of evidence). ${ }^{3235} 3739$ Adding three studies at high risk of bias did not change the result (risk ratio 0.92, 0.69 to 1.23). ${ }^{303340}$ More patients treated with a second generation antidepressant than receiving cognitive behavioral therapy withdrew from studies owing to adverse events (risk ratio 2.54, 0.39 to 16.47; three studies ${ }^{353739} ; \mathrm{I}^{2}=62 \%$ ), but the difference was not statistically significant (low strength of evidence). Again, including a trial at high risk of bias did not affect the results (risk ratio 2.97, 0.69 to 12.81$) .40$ Finally, second generation antidepressants and cognitive behavioral therapies did not differ significantly in terms of dropouts attributed to lack of efficacy in the main analysis (risk ratio $0.36,0.06$ to 2.21; three studies $^{353739}$; $\mathrm{I}^{2}=51 \%$ ).

Second generation antidepressant versus cognitive behavioral therapy: combination comparisons

The three trials that compared second generation antidepressant monotherapy with a combination of second generation antidepressant and cognitive behavioral therapy reported no statistically significant between group differences in rates of either remission or response (low strength of evidence). ${ }^{39} 4243$ All reported change in depression scale score between baseline and endpoint; one reported a significant between group difference-namely, a smaller decrease in scores on the
Montgomery-Åsberg Depression Rating Scale for patients taking second generation antidepressant alone than for patients treated with second generation antidepressant plus cognitive therapy. ${ }^{43}$ That trial, however, was rated as at high risk of bias, whereas the other two were rated as at low ${ }^{42}$ and medium ${ }^{39}$ risk of bias.

The trial that compared second generation antidepressant alone with second generation antidepressant plus telephone cognitive behavioral therapy measured several work related outcomes. ${ }^{42}$ Patients receiving the combination of second generation antidepressant and telephone cognitive behavioral therapy reported greater improvement on three of four work functioning measures. The authors found no between group differences in reduction of hours of work missed, although both groups reported a decrease at the end of treatment. In the trial that compared second generation antidepressant alone with the combination of second generation antidepressant and problem solving therapy, groups did not differ in the Social Adjustment Scale at end of treatment or at 40 week off-treatment follow-up. ${ }^{39}$

Two of the three trials reported adverse events. ${ }^{39} 42$ In the trial rated as at low risk of bias, more patients withdrew for any reason from the combination arm than from the second generation antidepressant alone arm (23\% v 13\%; significance not reported). ${ }^{42}$ However, slightly more patients withdrew owing to adverse events from the second generation antidepressant alone arm (6\%) compared with the combination arm (4\%). In the other trial, rated as at medium risk of bias, discontinuation rates were similar between the second generation antidepressant alone and combination arms (17\% in each arm). ${ }^{39}$ Eleven per cent and $6 \%$ of patients in the combination and second generation antidepressant arms, respectively, withdrew because of adverse events. No patients in either arm withdrew because of lack of treatment efficacy. The low number events in each of these outcomes results in a low strength of evidence rating.

\section{Discussion}

For second generation antidepressants compared with cognitive behavioral therapies, the available evidence based on 11 randomized controlled trials with 1511 patients suggests no difference in treatment effects of second generation antidepressants and cognitive behavioral therapies, either alone or in combination, although our conclusions are tempered by small numbers and mostly low strength of evidence. Relative risks of remission and response were nearly identical for monotherapy comparisons ( 0.98 and 0.91 , respectively) and for second generation antidepressant alone versus combination (1.06 and 1.03, respectively). Adverse event outcomes were also clinically and statistically similar for all comparisons.

\section{Comparison with other studies and guidelines}

Our findings are relatively consistent with similar meta-analyses by Cuijpers and colleagues that compared second generation antidepressants with 
cognitive behavioral therapy. ${ }^{2546-48}$ In addition to containing several more recent studies, our analyses have some key methodological differences that make them more relevant for the primary care population. Cuijpers and colleagues did meta-analyses of second generation antidepressants compared with any psychotherapy and of cognitive behavioral therapy compared with any drug, but they did not report results from a specific comparison between second generation antidepressant and cognitive behavioral therapy for adults with major depressive disorder. They found that only selective serotonin reuptake inhibitors, rather than second generation antidepressants as a class, were more effective than any type of psychotherapy in treating patients with major depressive disorder; however, the effect was small and potentially clinically insignificant. Furthermore, they did not require the use of evidence based psychotherapy (for example, non-directive supportive counseling was eligible for their analyses), they did not limit studies to those in which patients had not already failed a previous antidepressant treatment (who would be more likely to be seen in primary care). Finally, their analyses did not report on comparative harms, which we have done in this report.

Spielmans and colleagues found that bona fide cognitive behavioral therapy (based on their included studies' descriptions of therapist training, therapist-client relationship, and therapy components) resulted in better outcomes that second generation antidepressant, but the effect sizes were small and heterogeneity was high. ${ }^{25}$ Although we considered treatment fidelity in our risk of bias assessment, we did not attempt to determine whether the cognitive behavioral therapy offered in the included trials was "bona fide." Because primary care clinicians are unlikely to have the information necessary to determine whether the cognitive behavioral therapy to which they refer patients is bona fide, our results may be more relevant to primary care providers.

Our results are also consistent with the recommendations of the American Psychiatric Association, ${ }^{3}$ the US Department of Veterans Affairs/Department of Defense, ${ }^{49}$ and the UK National Institute for Health and Care Excellence. ${ }^{50}$ Those groups consider both drugs and psychotherapy to be appropriate individual initial treatments for patients with mild to moderate major depressive disorder. Furthermore, they state that drugs plus psychotherapy may be a useful initial treatment for patients with a current episode of moderate to severe major depressive disorder and for those with major depressive disorder and coexisting conditions.

\section{Limitations}

This review, and the evidence that informed it, does have limitations. The scope of this review was limited to trials that enrolled adult patients with major depressive disorder and compared a second generation antidepressant with cognitive behavioral therapy (alone or in combination with a second generation antidepressant). We did not attempt to review the literature on interventions for major depressive disorder in children or for patients with subthreshold depression, dysthymia, or perinatal depression. The included trials enrolled mostly patients with moderate to severe major depressive disorder; therefore, results may not be applicable to patients with milder major depressive disorder. Most trials excluded patients with medical comorbidities or suicidal ideation; few trials included elderly patients. We found insufficient evidence to confirm or refute whether treatments are more or less efficacious for various subgroups (patients characterized by sex, race, or ethnicity or those with coexisting psychiatric conditions).

Many of the included trials, even those rated as at medium risk of bias, had methodological shortcomings that may limit confidence in some of our findings. Several studies reported very high attrition, although rates were usually similar between treatment groups. Our "worst case" assumption of withdrawals as failures to respond or remit should alleviate some of that concern. Most of the included trials provided data only for acute phase treatment; information to help providers to manage ongoing depression or prevent relapse and recurrence is lacking.

Although studies of psychotherapy contain some inherent heterogeneity of the content and delivery, the included trials used treatment manuals with prescribed goals and techniques to minimize variability between therapists. The type, training, and experience of the clinicians who render these various interventions were also quite diverse. Unlike the case with second generation antidepressants, which are broadly equivalent and have standardized dosing, the cumulative effect of the various sources of heterogeneity within and across psychological interventions may limit the applicability of our findings. Clinician type, training, experience, and degree of treatment fidelity are likely to be even less consistent in routine clinical practice than in the trials in this review. Along with availability of psychotherapists, these are important factors for clinicians to consider when recommending psychological treatment and interventions.

Finally, comparative effectiveness at a group level did not detect a difference between second generation antidepressants and cognitive behavioral therapies, but how best to tailor this information to an individual patient is still not clear, especially given the low strength of evidence for most of the included outcomes. Preliminary evidence suggests that biomarkers may have a role in predicting response to cognitive behavioral therapy compared with second generation antidepressants, but these findings have yet to be replicated or implemented in clinical practice. ${ }^{30}$ Analyses of individual patient level data are best suited to answer that question and should be done in the future. Although we searched for unpublished literature, publication bias and selective outcome reporting may still be potential limiting factors. Finally, because we did not compare active treatment with placebo or no treatment, we cannot rule out the possibility that cognitive behavioral therapy and second generation antidepressants may be 
similarly ineffective. However, because evidence of benefit from both treatments exists, a provider would be unlikely recommend no treatment instead.

\section{Conclusions and policy implications}

In clinical decision making, providers should consider not only the efficacy of second generation antidepressants and cognitive behavioral therapy interventions but also patients' preferences about potential adverse events, the costs and availability of each treatment, and expected treatment effects. Currently, the biggest barrier to offering psychotherapy either alone or in combination with drugs may be how well patients can gain access to such mental healthcare clinicians. Given that the benefits of second generation antidepressants and cognitive behavioral therapy do not seem to differ significantly in treating major depressive disorder and that primary care patients may have personal preferences for one first line treatment over the other, both treatments should be made accessible, either alone or in combination, to primary care patients with major depressive disorder. ${ }^{8}$

Having access to psychotherapeutic interventions in the primary care setting might improve treatment outcomes for patients with major depressive disorder. It has the potential to improve use of psychiatric consultations and therapy and to enhance coordination of care between primary care clinicians and mental health professionals. It may also have additional downstream effects of reducing the stigma associated with mental illness in general, empowering patients to tackle the symptoms and problems associated not only with depression but also with other mental health related concerns and encouraging them to seek and maintain treatment more quickly at an earlier stage of their illness.

We thank Meera Viswanathan and Loraine Monroe, both from RTI International, for dedicated support and Irma Klerings from Danube University, Krems, for literature searches.

Contributors: HRA reviewed studies for inclusion and exclusion; entered, cleaned, and analyzed the data; did statistical analyses; and drafted and revised the paper. GG oversaw the design and conduct of the full report from which this manuscript was devised, reviewed included studies, analyzed data, and revised the draft paper. BNG, CF, GNA, LCM, EC-S, EB, LJL, SG, CB, and CBP participated fully in the report from which this manuscript was devised, reviewed included studies, entered and cleaned data, and revised the draft paper. BNG, LCM, and CB also did data analyses. KNL contributed to the full report from which this project was based and revised the draft paper. HRA is the guarantor.

Funding: This project was funded under contract 290-2012-00008i from the Agency for Healthcare Research and Quality by the RTI-UNC Evidence-based Practice Center. The authors of this manuscript are responsible for its content. Statements in this manuscript should not be construed as endorsement by the Agency for Healthcare Research and Quality or the US Department of Health and Human Services.

Competing interests: The authors have completed the ICMJE uniform disclosure form at www.icmje.org/coi_disclosure.pdf (available on request from the corresponding author) and declare: no support from any organization other than the funding agency listed above for the submitted work; no financial relationships with any organizations that might have an interest in the submitted work in the previous three years; no other relationships or activities that could appear to have influenced the submitted work.

Ethical approval: Not needed for this project.

Data sharing: Detailed methods and additional information are available in the full report, available at http://effectivehealthcare.ahrq.gov/.
Transparency: The lead author (the manuscript's guarantor) affirms that this manuscript is an honest, accurate, and transparent account of the study being reported; that no important aspects of the study have been omitted; and that any discrepancies from the study as planned (and, if relevant, registered) have been explained.

This is an Open Access article distributed in accordance with the Creative Commons Attribution Non Commercial (CC BY-NC 4.0) license, which permits others to distribute, remix, adapt, build upon this work non-commercially, and license their derivative works on different terms, provided the original work is properly cited and the use is non-commercial. See: http://creativecommons.org/licenses/ by-nc/4.0/.

1 Kessler RC, Berglund P, Demler O, et al. The epidemiology of major depressive disorder: results from the National Comorbidity Survey Replication (NCS-R). JAMA 2003;289:3095-105.

2 Wang PS, Lane M, Olfson M, et al. Twelve-month use of mental health services in the United States: results from the National Comorbidity Survey Replication. Arch Gen Psychiatry 2005;62:629-40.

3 American Psychiatric Association (APA). Practice guideline for the treatment of patients with major depressive disorder. 3rd ed. APA, 2010.

4 Qaseem A, Snow V, Denberg TD, et al. Using second-generation antidepressants to treat depressive disorders: a clinical practice guideline from the American College of Physicians. Ann Intern Med 2008; 149:725-33

5 Institute of Medicine. Crossing the quality chasm: a new health system for the 21st century. National Academies Press, 2001

6 Mojtabai R, Olfson M. National trends in long-term use of antidepressant medications: results from the U.S. National Health and Nutrition Examination Survey. J Clin Psychiatry 2014;75:169-77.

7 Gartlehner G, Hansen RA, Morgan LC, et al. Comparative benefits and harms of second-generation antidepressants for treating major depressive disorder: an updated meta-analysis. Ann Intern Med 2011;155:772-85.

8 Xing S, Dipaula BA, Lee HY, et al. Failure to fill electronically prescribed antidepressant medications: a retrospective study. Prim Care Companion CNS Disord 2011;13:pii: PCC.10m00998.

9 Gartlehner G, Thieda P, Hansen RA, et al. Comparative risk for harms of second-generation antidepressants: a systematic review and meta-analysis. Drug Saf 2008:31:851-65.

10 McHugh RK, Whitton SW, Peckham AD, et al. Patient preference for psychological vs pharmacologic treatment of psychiatric disorders: a meta-analytic review. I Clin Psychiatry 2013:74:595-602.

11 Raue PJ, Schulberg HC, Heo M, et al. Patients' depression treatment preferences and initiation, adherence, and outcome: a randomized primary care study. Psychiatr Serv 2009;60:337-43.

12 Churchill R, Khaira M, Gretton V, et al. Treating depression in general practice: factors affecting patients' treatment preferences. BrJ Gen Pract 2000;50:905-6.

13 Dwight-Johnson M, Sherbourne CD, Liao D, et al. Treatment preferences among depressed primary care patients. J Gen Intern Med 2000;15:527-34

14 Brody DS, Khaliq AA, Thompson TL 2nd. Patients' perspectives on the management of emotional distress in primary care settings. J Gen Intern Med 1997;12:403-6.

15 Morey E, Thacher JA, Craighead WE. Patient preferences for depression treatment programs and willingness to pay for treatment. $J$ Ment Health Policy Econ 2007;10:73-85.

16 Priest RG, Vize C, Roberts A, et al. Lay people's attitudes to treatment of depression: results of opinion poll for Defeat Depression Campaign just before its launch. BMJ 1996;313:858-9.

17 Van Schaik DJ, Klijn AF, van Hout HP, et al. Patients' preferences in the treatment of depressive disorder in primary care. Gen Hosp Psychiatry 2004;26:184-9.

18 Wittink MN, Cary M, Tenhave T, et al. Towards patient-centered care for depression: conjoint methods to tailor treatment based on preferences. Patient 2010:3:145-57.

19 Cooper LA, Gonzales JJ, Gallo JJ, et al. The acceptability of treatment for depression among African-American, Hispanic, and white primary care patients. Med Care 2003;41:479-89.

20 Givens JL, Houston TK, Van Voorhees BW, et al. Ethnicity and preferences for depression treatment. Gen Hosp Psychiatry 2007;29:182-91.

21 Mergl R, Henkel V, Allgaier AK, et al. Are treatment preferences relevant in response to serotonergic antidepressants and cognitive-behavioral therapy in depressed primary care patients? Results from a randomized controlled trial including a patients' choice arm. Psychother Psychosom 2011;80:39-47.

22 Cuijpers P, van Straten A, Andersson G, et al. Psychotherapy for depression in adults: a meta-analysis of comparative outcome studies. J Consult Clin Psychol 2008;76:909-22.

23 Nieuwsma JA, Trivedi RB, McDuffie J, et al. Brief psychotherapy for depression: a systematic review and meta-analysis. Int J Psychiatry Med 2012;43:129-51. 
24 American Psychological Association. Recognition of psychotherapy effectiveness. 2012. www.apa.org/about/policy/resolutionpsychotherapy.aspx

25 Spielmans GI, Berman MI, Usitalo AN. Psychotherapy versus second-generation antidepressants in the treatment of depression: a meta-analysis. J Nerv Ment Dis 2011;199:142-9.

26 Gartlehner G, Gaynes B, Amick H, et al. Nonpharmacological versus pharmacological treatments for adult patients with major depressive disorder. Agency for Healthcare Research and Quality, 2015

(Comparative Effectiveness Review No 161). www.effectivehealthcare ahrq.gov/reports/final.cfm.

27 Cochrane Depression, Anxiety and Neurosis Group. CCDAN topic list: intervention-psychological therapies. 2013 http://cmd cochrane. org/sites/cmd.cochrane.org/files/uploads/CCDAN\%20topics\%20 list_psychological\%20therapies\%20for\%20website_0.pdf

28 Higgins IP, Altman DG, Gotzsche PC, et al. The Cochrane Collaboration's tool for assessing risk of bias in randomized trials. $B M$ 2011;343:d5928.

29 Berkman ND, Lohr KN, Ansari MT, et al. Grading the strength of a body of evidence when assessing health care interventions: an EPC update. J Clin Epidemiol 2015;68:1312-24.

30 McGrath CL, Kelley ME, Holtzheimer PE, et al. Toward a neuroimaging treatment selection biomarker for major depressive disorder. JAMA Psychiatry 2013;70:821-9.

31 Sava FA, Yates BT, Lupu V, et al. Cost-effectiveness and cost-utility of cognitive therapy, rational emotive behavioral therapy, and fluoxetine (Prozac) in treating depression: a randomized clinical trial. / Clin Psychol 2009;65:36-52.

32 David D, Szentagotai A, Lupu V, et al. Rational emotive behavior therapy, cognitive therapy, and medication in the treatment of major depressive disorder: a randomized clinical trial, posttreatment outcomes, and six-month follow-up. J Clin Psychol 2008;64:728-46.

33 Kennedy SH, Konarski JZ, Segal ZV, et al. Differences in brain glucose metabolism between responders to cognitive behavioral therapy and venlafaxine in a 16-week randomized controlled trial. Am J Psychiatry 2007;164:778-88

34 Leykin Y, Amsterdam JD, DeRubeis RJ, et al. Progressive resistance to a selective serotonin reuptake inhibitor but not to cognitive therapy in the treatment of major depression. J Consult Clin Psychol 2007;75:267-76

35 Dimidjian S, Hollon SD, Dobson KS, et al. Randomized trial of behavioral activation, cognitive therapy, and antidepressant medication in the acute treatment of adults with major depression. J Consult Clin Psychol 2006;74:658-70.

36 Segal ZV, Kennedy S, Gemar M, et al. Cognitive reactivity to sad mood provocation and the prediction of depressive relapse. Arch Gen Psychiatry 2006;63:749-55.

37 DeRubeis RJ, Hollon SD, Amsterdam JD, et al. Cognitive therapy vs medications in the treatment of moderate to severe depression. Arch Gen Psychiatry 2005;62:409-16
38 Miranda J, Chung JY, Green BL, et al. Treating depression in predominantly low-income young minority women: a randomized controlled trial. JAMA 2003;290:57-65.

39 Mynors-Wallis LM, Gath DH, Day A, et al. Randomised controlled trial of problem solving treatment, antidepressant medication, and combined treatment for major depression in primary care. $B M$ 2000;32:26-30.

40 Hegerl U, Hautzinger M, Mergl R, et al. Effects of pharmacotherapy and psychotherapy in depressed primary-care patients: a randomized, controlled trial including a patients' choice arm. Int I Neuropsychopharmacol 2010;13:31-44.

41 Landenberger NAD. Self-concept and attributional style in the treatment of depression. ProQuest Information and Learning, 2002.

42 Lam RW, Parikh SV, Ramasubbu R, et al. Effects of combined pharmacotherapy and psychotherapy for improving work functioning in major depressive disorder. Br J Psychiatry 2013;203:358-65.

43 Shamsaei F, Rahimi A, Zarabian MK, et al. Efficacy of pharmacotherapy and cognitive therapy, alone and in combination in major depressive disorder. Hong Kong J Psychiatr 2008;18:76-80.

44 Berkman ND, Lohr KN, Ansari M, et al. Grading the strength of a body of evidence when assessing health care interventions for the effective health care program of the agency for healthcare research and quality: an update. 2103. In: Methods guide for effectiveness and comparative effectiveness reviews [Internet]. Agency for Healthcare Research and Quality (US), 2008 (available at www.ncbi.nlm.nih.gov/ books/NBK174881/)

45 Dobson KS, Hollon SD, Dimidjian S, et al. Randomized trial of behavioral activation, cognitive therapy, and antidepressant medication in the prevention of relapse and recurrence in major depression. J Consult Clin Psychol 2008;76:468-77.

46 Cuijpers P, Berking M, Andersson G, et al. A meta-analysis of cognitive-behavioural therapy for adult depression, alone and in comparison with other treatments. Can J Psychiatry 2013;58:376-85.

47 Cuijpers P. Sijbrandii M, Koole SL, et al. The efficacy of psychotherapy and pharmacotherapy in treating depressive and anxiety disorders: a meta-analysis of direct comparisons. World Psychiatry 2013;12:137-48

48 Cuijpers P, Sijbrandij M, Koole SL, et al. Adding psychotherapy to antidepressant medication in depression and anxiety disorders: a meta-analysis. World Psychiatry 2014;13:56-67.

49 Department of Veterans Affairs, Department of Defense. VA/DoD clinical practice guideline for management of major depressive disorder (major depressive disorder). Department of Veterans Affairs, Department of Defense, 2009.

50 National Institute for Health and Care Excellence (NICE). Depression in adults: recognition and management.NICE, 2009 (clinical guideline CG90) (available from www.nice.org.uk/guidance/cg90). 